\title{
Priority Parameter-based Analysis and Optimization for EDCA
}

\author{
Zhen Wang and Xiao Guo \\ School of Information Engineering, Communication University of China \\ Beijing, China \\ wz9921@163.com,xguo@cuc.edu.cn
}

\begin{abstract}
This article aims at coming up the optimization of the throughput network performance in EDCA. At first, we observe in different network load conditions that the effect of each priority parameter has on the network transmission performance. Then we make a deep research about the EDCA mechanism and come up a new EDCA analysis model and there is a throughput analysis of this model and a optimization scheme of the priority business flows under different network loads. Finally, based on the mathematical analysis and NS2 simulation results, it can be observed from the new optimization scheme that it to some extent improves different priority services under different network loads.
\end{abstract}

Keywords: EDCA; Priority parameter; Analysis model; Throughput

\section{Introduction}

The IEEE 802.11-based wireless local area network has been widely used these years. It provides us two access models: PCF (Point Coordinate Function) and DCF (Distributed Coordinate Function) which however could not have a good support of the quality of service. While IEEE802.11e introduces HCF (Hybrid Coordinate Function) and EDCA mechanism to replace two kinds of channel access modes of the PCF and DCF in the IEEE802.11 to guarantee quality of service [1].

This article aims to analyze the IEEE 802.11e-proposed EDCA mechanism. EDCA introduces a different type of access and the priority parameters, so as to realize service differentiation and come up the optimization scheme based on mathematical model.

EDCA introduces 4 ACs (Access Categories) in which each category has its own priority parameter (such as AIFS(Arbitration Inter-frame Space ), CW_min(Minimum Contention Window), CW_max(Maximum Contention Window )and TXOP(Transmission Opportunity Limit) over other access categories as shown in Figure 1.

Based on the above three priority parameters, this article tries to make a simulation analysis of the affects of the EDCA performance and aims at putting forward the network performance optimization of EDCA. The second part of this article comes up the simulation program [2]. The third part attempts to analyze different priority parameters under different network loads. In the fourth part, proposes a new IEEE 802.11e network analysis model and comes out its mathematical expressions of the throughput and to testify the validity of the optimization with the help of simulation. 


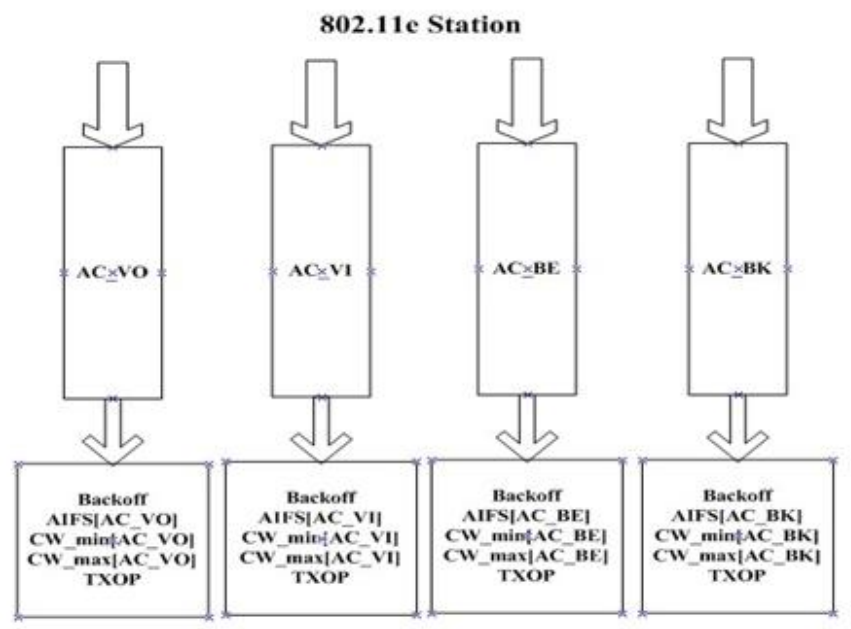

Figure 1. EDCA backoff entity

\section{Simulation Model}

Simulation in this paper uses a simulation scene in which the wired network and wireless network mixed. Such hybrid network includes an access point AP, a cable station and 4 wireless stations [3]. The base station and cable station are connected by the bidirectional wired link, and the link bandwidth is $10 \mathrm{M} / \mathrm{s}$, while the wireless station is linked with the stations by wireless transmission, and the bandwidth is $1 \mathrm{Mb} / \mathrm{s}$. Besides, the communication between the wireless station and the wired station as well as the communication among different wireless stations should be transited by the base station.

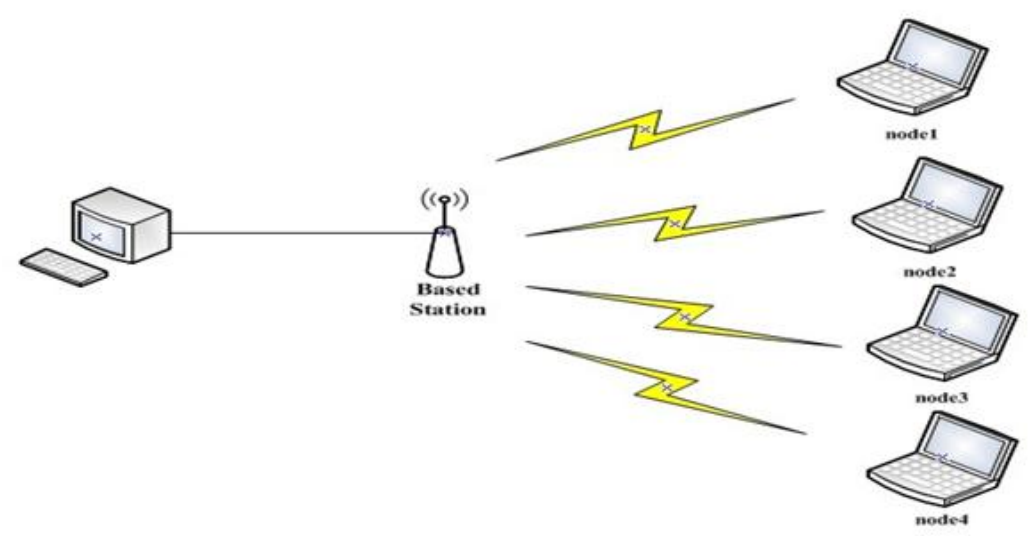

Figure 2. Simulation model

As shown in Figure 2, each site sends $\mathrm{AC} 0, \mathrm{AC} 1, \mathrm{AC} 2$, and $\mathrm{AC} 3$ these four kinds of business flow. Among these four business flows, AC0 represents the real-time business flow, and $\mathrm{AC} 2$ represents the common data flow. We first assign different parameter values to the AIFS, CW_min, CW_max and TXOP, then observe the influence of EDCA's various priority parameters over the network performance under some circumstances such as the increased station sending rate, overloaded network load and the increasing channel competition [4]. Then we build up mathematical model and analyze the throughput of the node under different 
network circumstances to verify the influence of priority parameter on network performance, and propose a reasonable optimization scheme of network performance.

\section{Simulation Results and Performance Comparison}

In this part we attempts to analyze different priority parameters under different network loads, and compare the simulation results to find out that how priority parameters have significant effect on the network performance .In this simulation, $x$-axis is time (s), y-axis is throughput(bytes).

\subsection{AIFS}

In scheme 1, we adopt the IEEE802.11e defaulted AIFS parameter value, and as shown in table 1, the AIFS parameter values are changed in scheme 2 and scheme 3 . Then we compare the $\mathrm{AC} 0$ and $\mathrm{AC} 2$ data flow simulation results in these three schemes under the increasing network load conditions.

Table 1. AIFS parameter

\begin{tabular}{|l|c|c|c|c|c|}
\hline & & AC0(VO) & AC1 & AC2(BE) & AC3 \\
\hline Scheme 1 & AIFS & 2 & 2 & 3 & 7 \\
\hline Scheme 2 & AIFS & 2 & 4 & 6 & 10 \\
\hline Scheme 3 & AIFS & 1 & 2 & 3 & 7 \\
\hline
\end{tabular}

Figure 3 clearly shows that the throughput of the real-time business in scheme 1 is lower than that in scheme 2 and scheme 3, while the throughput of the data flow is higher. The reason is that compared with scheme 1, the data flow in scheme 2 has higher AIFS, so the data flow has less priority to access the channel [5]. While the real time business flow in scheme 3 has lower AIFS, so the business flow has more priority to access the channel. From the throughput of the real time business flow, we can draw conclusions that either increasing the AIFS with lower priority or reducing the AIFS with higher priority can improve the performance of the business flow with high priority.

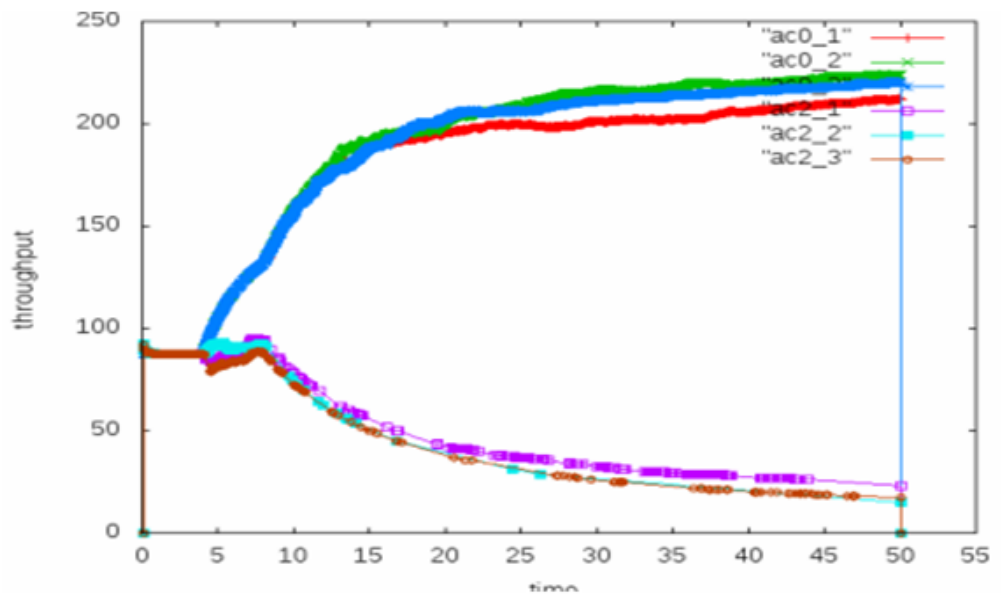

Figure 3. AlFS simulation 


\subsection{CW_min And CW_max}

In scheme 1, we adopt the IEEE802.11e defaulted CW_min and CW_max parameter value, and as shown in Table 2, the CW_min and CW_max parameter value are changed in scheme 2. Then we compare the AC0 and AC2 data flow simulation results in these three schemes under the increasing network load conditions.

Table 2. CW parameter

\begin{tabular}{|c|c|c|c|c|}
\hline Scheme 1 & AC0(VO) & AC1 & AC2(BE) & AC3 \\
\hline CW_min & 7 & 15 & 31 & 31 \\
\hline CW_max & 15 & 31 & 1023 & 1023 \\
\hline Scheme 2 & AC0(VO) & AC1 & AC2(BE) & AC3 \\
\hline CW_min & 7 & 15 & 256 & 511 \\
\hline CW_max & 15 & 31 & 1023 & 1023 \\
\hline
\end{tabular}

Figure 4 obviously shows that when it is in low load condition, the contention window of the data flow becomes larger, and the data access latency is longer. But because the system is in the low load condition, so the channel competition is not fierce, and the throughput of the real-time business flow increases not so significantly. In contrast, because of the increased data access latency, the throughput of the data flow decreases [6]. When the data transmission rate becomes larger, the system is more and more heavy-loaded. In Figure 4, the throughput of the real-time business flow in scheme 1 is lower than that in scheme 2 , and the throughput of the data flow in scheme 1 is higher than that in scheme 2 . When the contention window of the data flow becomes larger, the data flow access latency will become longer. So the realtime business flow acquires higher priority access channel and the throughput increases.

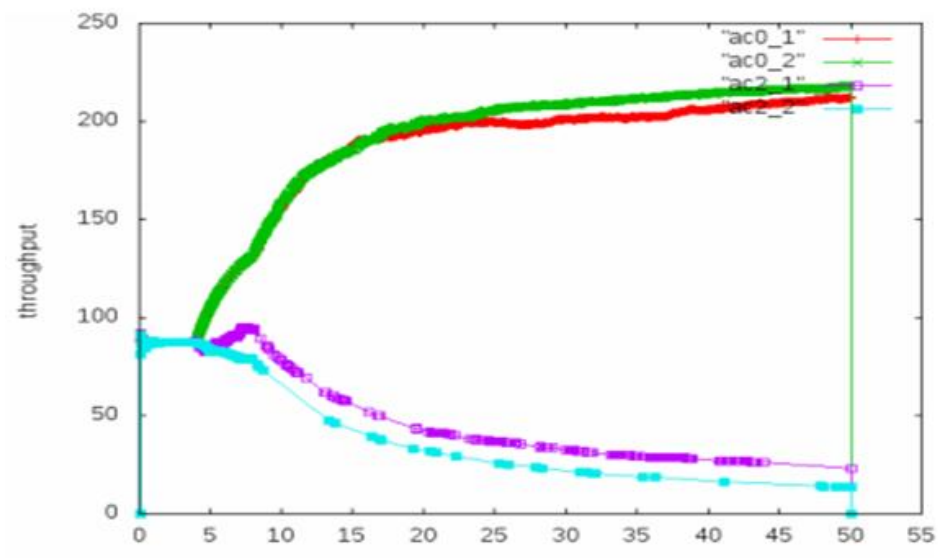

Figure 4. CW simulation

\subsection{TXOP}

In scheme 2, we adopt the IEEE802.11e defaulted TXOP parameter value, and as shown in Table 3, the TXOP parameter value are changed in scheme 1 and scheme 3 . Then we compare the $\mathrm{AC} 0$ and $\mathrm{AC} 2$ data flow simulation results in these three schemes under the increasing network load conditions. 
Table 3. TXOP parameter

\begin{tabular}{|l|c|c|c|c|c|}
\hline & & AC0(VO) & AC1 & AC2(BE) & AC3 \\
\hline Scheme 1 & TXOP & 0 & 0 & 0 & 0 \\
\hline Scheme 2 & TXOP & 0.003264 & 0.006016 & 0 & 0 \\
\hline Scheme 3 & TXOP & 0.006016 & 0.006016 & 0 & 0 \\
\hline
\end{tabular}

From Figure 5 we can see that scheme 1 does not invoke TXOP, so every time it acquires the channel, it can send one frame only. Scheme 2 is the 802.11 e defaulted TXOP value. Scheme 3 is the improved the real-time business flow TXOP value. In scheme 2 and scheme 3 , the station can send multiple frames every time when it has the opportunity to gain the channel instead of regaining the new channel to send the next frame. But as can be seen from the graph, the throughput curves of the three schemes' real-time business flow are almost identical, which indicates that the increase of TXOP value has limit improvement on the network performance [7]. Comparing the simulation of different TXOP values with the simulation of different contention window CW and AIFS, it can be found that the TXOP curve of discrimination is much less than that of the CW and AIFS value, namely, to increase the TXOP value rather than the CW and AIFS values, though the system performance is enhanced, it is not so obvious. As a result, it shows that compared with other parameters, the increase of the TXOP value improves little on the system performance.

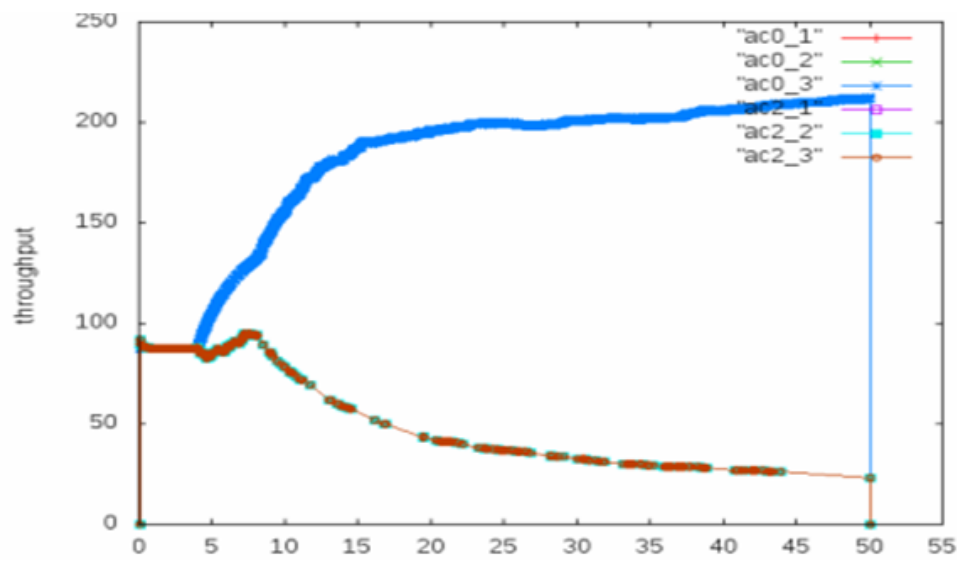

Figure 5. TXOP simulation

\section{Analysis of IEEE 802.11e Network Model}

This part can be divided into two parts. The first part is to establish a new IEEE 802.11e network analysis model, then to analyze the internal site data transmit process and make an abstract description of the contention and transmission of different priority services with the help of mathematical model. According to the results of the first part, the second part is to establish the throughput analysis model and get the mathematical expressions of throughput and then put forward a new optimization scheme [8].

\subsection{Markov Model}

Based on the previously proposed 802.11DCF access mode network analysis model, a new 802.11e network analysis model is multilevel-extended and proposed. This is a model based 
on the site competition, and it considers the effect of site internal scheduling algorithm on multi-business competition.

Suppose there are $\mathrm{n}$ sites, each site is in the saturated state and has $\mathrm{N}$ priority services. $\mathrm{s}(\mathrm{i}, \mathrm{t})$ denotes the back off order of the $i$ access categories, $b(i, t)$ is the backoff counter of the $i$ access categories. P1 indicates the channel error probability of the $\mathrm{i}$ access categories transmission. $\mathrm{P} 2$ is the conflict probability of the data transmission when the back timer turns to $0 . \mathrm{P} 3$ shows the probability of the busy channel as the $\mathrm{i}$ access categories in the channel contention. Similar to the previous network model, the random process (i, s (i, t), b (i, t)) can be regarded as a 2D Markov [9] chain is shown in Figure 6.

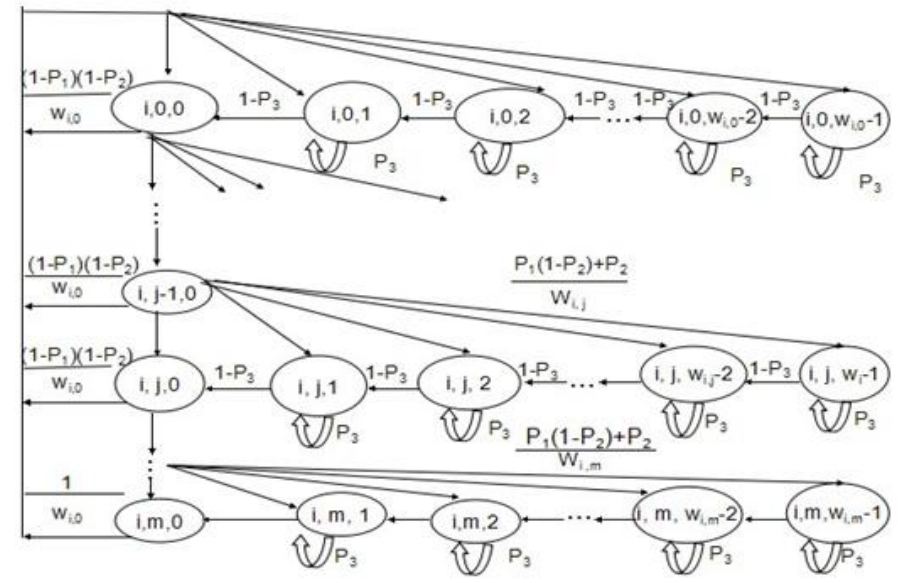

Figure 6. Markov model

The slot can be the idle time slot, slot that with successful data packet transmission, collision slots or slot that with channel transmission error [10]. For a particular state (i, j, k), every time the backoff counter comes across an idle time slot, the probability of the value of one less is 1-P3. While if the channel is busy when it is retreated, the frozen probability of the backoff counter is P3. But if there is a packet conflict or transmission error, the retransmission counter value will plus 1 , and it will randomly access into the backoff process in the next layer with the probability of $[\mathrm{P} 1(1-\mathrm{P} 2)+\mathrm{P} 2] / \mathrm{Wi}, \mathrm{j}$. If the data packet is successfully transferred, the probability will be $[(1-\mathrm{P} 1)(1-\mathrm{P} 2)] / \mathrm{Wi}, 0$, and the sequential packet sending will start a new round of competition from the zeroth order. If the maximum limit of the retransmissions is reached, whether the data is transmitted successfully or not, the retransmission counter's values are cleared, and will be transferred to zero order with the probability of $1 / \mathrm{Wi}, 0$. So the Markov chain can be described as in formula (1).

$$
\left\{\begin{array}{l}
P\{i, j, k \mid i, j, k+1\}=1-P_{3} \quad k \in\left\{0, W_{i, j}-2\right\} ; j \in\{0, m\} \\
P\{i, j, k \mid i, j, k\}=P_{3} \quad k \in\left\{0, W_{i, j}-1\right\} ; j \in\{0, m\} \\
P\{i, j, k \mid i, j-1, k\}=\left[P_{1}\left(1-P_{2}\right)+P_{2}\right] / W_{i, j} \quad k \in\left\{0, W_{i, j}-1\right\} ; j \in\{0, m\} \\
P\{i, j, k \mid i, j-1, k\}=\left[\left(1-P_{1}\right)\left(1-P_{2}\right)\right] / W_{i, 0} \quad k \in\left\{0, W_{i, j}-1\right\} ; j \in\{0, m\} \\
P\{i, 0, k \mid i, m, 0\}=1 / W_{i, 0} \quad k \in\left\{0, W_{i, j}-1\right\}
\end{array}\right.
$$

In formula (1), Wi,j indicates the size of the contention window when the $\mathrm{i}$ type business is in the number $\mathrm{j}$ backoff order, and $\mathrm{m}$ is the maximum number of retransmissions, $\mathrm{m}$ ' denotes 
the maximum number of the backoff order [11]. The relation between Wi,j and Wi,0is shown as in formula (2).

$$
W_{i, j}= \begin{cases}2^{j} W_{i, 0} & j \in\left[\mathrm{O}, m^{\prime}\right] \\ 2^{m^{\prime}} W_{i, 0} & j \in\left[m^{\prime}, m\right]\end{cases}
$$

Suppose pi is the probability of data trames sending from the number i type business to the given time slot, while $\phi \mathrm{T}$ is the data sending probability of the site in this time slot [12].

$$
\left\{\begin{array}{l}
a_{i, j, k}=\lim _{t \rightarrow>\infty} P\{s(i, t)=j, b(i, t)=k\} \\
\sum_{j=0}^{m} \sum_{k=0}^{W_{i, j-1}} a_{i, j, k}=1 \\
a_{i, j, k}=\frac{W_{i, j}-k}{W_{i, j}} \frac{1}{1-P_{3}} a_{i, j, 0} \\
a_{i, j, 0}=\left[P_{1}\left(1-P_{2}\right)+P_{2}\right]^{j} a_{i, 0,0} \\
\phi_{i}=\sum_{j=0}^{m} a_{i, j, 0} \\
\phi_{T}=\phi_{0}+\phi_{1}\left(1-\phi_{0}\right)+\cdots+\phi_{N-1} \coprod_{j=0}^{N-2}\left(1-\phi_{j}\right)
\end{array}\right.
$$

$\phi 1$, 1.e tne aata senaing probabilty or tne site in tnis time siot, can be arawn trom tormula (3).

\subsection{Throughput Model}

In the EDCA access mode, the slot length of a sending process means not the standard slot length, but should also includes time of successful transmission, conflict and waiting time when there is a channel transmission error [13].

Suppose Ps, i is the successful sending probability from the number i type business to the given slot, Ps means the total successful sending probability of site to given slot. Ts indicates the time it needed to send the data frame successfully. This can be shown in formula (4).

$$
\left\{\begin{array}{l}
P_{s i}=n \phi_{i}\left(1-P_{1}\right)\left(1-P_{2}\right) \\
P_{S}=\sum_{i=0}^{N-1} P_{s i} \\
T_{S}=T_{H}+T_{E(L)}+S I F S+\delta+A C K+A I F S[i]+\delta
\end{array}\right.
$$

Suppose Pe, $\mathrm{i}$ is the error sending probability from the number i type business to the given slot, PE means the total error sending probability of this site to the given slot. TE indicates the time it needed when there is a channel error [13]. This can be shown in formula (5).

$$
\left\{\begin{array}{l}
P_{e i}=n \phi_{i} P_{1}\left(1-P_{2}\right) \\
P_{E}=\sum_{i=0}^{N-1} P_{e i} \\
T_{E}=T_{H}+T_{E(L)}+A I F S[i]+\delta
\end{array}\right.
$$


Suppose Po is the free probability of the given time channel, and to indicates the waiting time of the free slot. This can be seen from formula (6).

$$
\left\{\begin{array}{l}
P_{O}=\left(1-\phi_{r}\right)^{n} \\
T_{O}=\theta
\end{array}\right.
$$

Suppose $\mathrm{P}_{\mathrm{c}}$ is the conflicting sending probability to the given time channel, and $\mathrm{T}_{\mathrm{c}}$ means the waiting time when there is a conflicting. This can be seen from formula (7).

$$
\left\{\begin{array}{l}
P_{C}=1-P_{S}-P_{E}-P_{O} \\
T_{C}=T_{H}+T_{E\left(L^{*}\right)}+A I F S[i]+\delta
\end{array}\right.
$$

In this article, what we discussed is the basic accessing model, and the results can be applied into RTS/CTS directly [14]. So the saturation throughput of the individual priority is :

$$
S_{i}=\frac{P_{s i} T_{E}(L)}{P_{O} \theta+\sum_{i=0}^{N-1} P_{s i} T_{S}+\sum_{i=0}^{N-1} P_{e i} T_{E}+\left(1-P_{S}-P_{E}-P_{O}\right) T_{C}}
$$

According to the formula (8), we get the expression of a single priority throughput. It is observed that the throughput is associated with four times, in which the occupied time by channel transmission error, the time delayed by failed transmission according to the conflict and the idle slot by channel contention are ineffective times. As the denominator of the formula, the new scheme should reduce these three times to improve network throughput [15]. In EDCA, the quality of service and the network performance are jointly determined by AIFS, CW_min, CW_max and other priority parameters, so we can adjust priority parameters to reduce these three ineffective times so as to improve the network throughput.

The time that wasted in the idle slot or occupied by the transmission error or that wasted caused by conflicting are of reciprocal relations, i.e., they could not increase or decrease simultaneously [16]. Besides, the network load is a dynamic one that we should adjust the priority parameters of each priority business according to the network load so as to improve the throughput of the individual priority. When it is in the low load condition, the conflicting probability in the data transmission is small, and it is better to reduce the wasted time in the idle slot and the time wasted in the failed channel transmission. Despite to guarantee the service quality, it's better to reduce the AIFS value of the higher priority as well as the CW_min and CW_max values of the lower priority. By doing this, the above two times are reduced and it improves the throughput of both the lower priority and the higher priority [17]. While when it is in the high load condition, the conflicting probability in the data transmission is large, and it is better to reduce the occupied time that caused by conflicting.It is advised to increase the AIFS value and the CW_min and CW_max values for it's hard for the lower priority service flow to seize the channels in high load condition, so it has no much effect on the throughput of the low priority even its AIFS and CW_min and CW_max values are increased, instead, because of the lower conflicting probability, the throughput of the higher priority will be much improved as shown in Figure 7. 


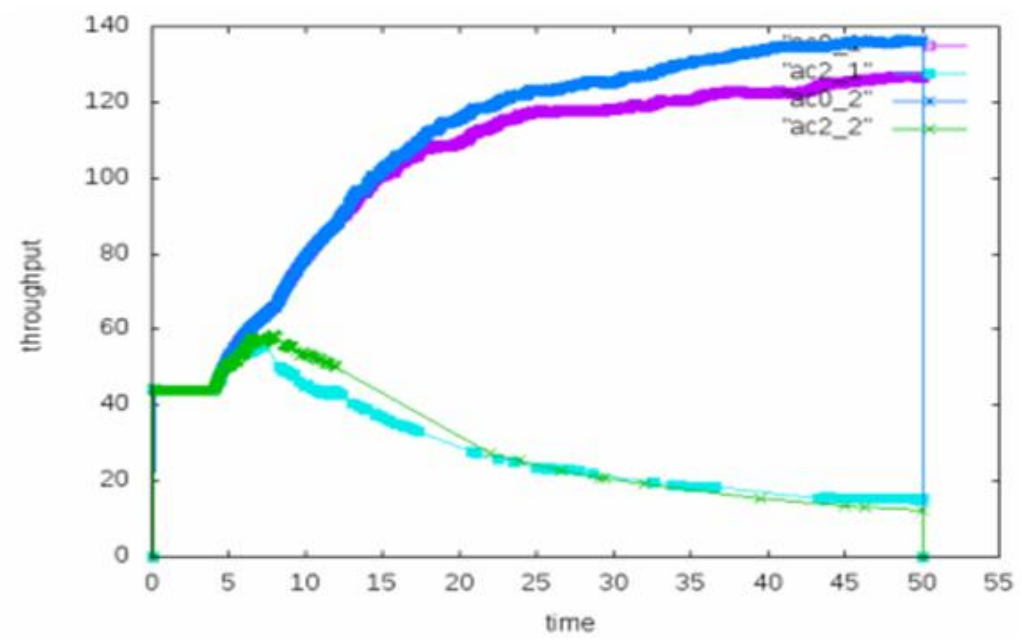

Figure 7. Optimization simulation

\section{Conclusion}

This article discussed the EDCA channel access mechanism in IEEE 802.11e. Based on the effect of internal scheduling algorithm on the multi-service competition, a new 2D Markov analysis model is proposed. Based on this model, we come up an analysis model of the network throughput of the EDCA as well as the expressions of the throughput of each priority business. Then according to the real dynamic network environment, by adjusting the priority parameters of different business flows in EDCA, an optimization scheme is proposed to optimize the throughput of different business flows. Finally, the simulation results show that the modified scheme is reasonable and feasible.

It is hoped that we can put forward the network delay model and jitter model to better improve the network performance and the quality of service of EDCA.

\section{Acknowledgements}

First, I would like to show my deepest gratitude to my supervisor Xiao Guo who gave me enlightening instruction in every stage of this paper. Second, I shall thank the professors and friends in Communication University of China for their useful suggestions. Last, thanks for my family for their persistent encouragement.

\section{References}

[1] I. Inan, F. Keceli and E. Ayanoglu, J. Communications, vol. 6, no. 57, (2009).

[2] J. Y. Lee and H. S. Lee, J. Communications, vol. 1, no. 57, (2009).

[3] R. J. Viegas, L. A. Guedes and F. G. Vasques, Computers \& Electrical Engineering, (2012).

[4] M. V. R. Azhaguramyaa, S. J. K. J. Kumar and P. Parthasarathi, Global Journal of Computer Science and Technology, vol. 5, no. 12, (2012).

[5] X. Pérez-Costa and D. Camps-Mur, Wireless Communications, vol. 4, no. 17, (2012).

[6] S. Rashwand and J. Misic, Vehicular Technology, vol. 3, no. 60, (2011).

[7] I. Tinnirello and G. Bianchi, NetworkinG, vol. 2, no. 18, (2010).

[8] G. Bianchi, IEEE, Journal Selected Areas in Communication, vol. 3, no. 18, (2000).

[9] H. Zhu and I. Chlamtac, Wireless Communications, vol. 4, no. 4, (2005).

[10] J. Hui and M. Devetsikiotis, Communications, vol. 9, no. 53, (2003).

[11] Y. Xiao, Wireless Communications, vol. 4, no. 4, (2005). 
[12] X. Chen, H. Zhai H, and X. Tian, Wireless Communications, vol. 8, no. 5, (2006).

[13] A. Banchs and L. Vollero, Computer Networks, vol. 11, no. 50, (2006).

[14] A. Hamidian and U. Körner, Telecommunication Systems, vol. 2, no. 31, (2006).

[15] P. Serrano, A. Banchs and P. Patras, Vehicular Technology, vol. 5, no. 59, (2010).

[16] W. Zhang, J. Sun and J. Liu, Journal of Zhejiang University SCIENCE A, vol. 1, no. 8, (2007).

[17] P. Patras, A. Banchs and P. Serrano, Mobile Networks and Applications, vol. 6, no. 14, (2009).

\section{Authors}

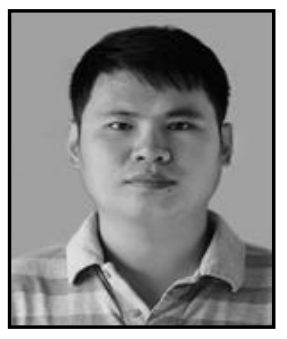

\section{Zhen Wang}

Male, postgraduate in Communication University of China, research on wireless QoS and next generation wireless network.

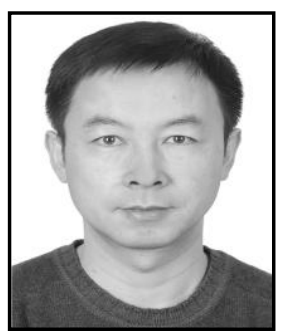

\section{Xiao Guo}

Male, Senior Engineer in Communication University of China, research on TCP/IP, wireless network. 